TITLE:

\title{
Time-resolved fluctuation during the photochemical reaction of a photoreceptor protein: Phototropin1LOV2-linker
}

\section{AUTHOR(S):}

Kuroi, Kunisato; Sato, Francielle; Nakasone, Yusuke; Zikihara, Kazunori; Tokutomi, Satoru; Terazima, Masahide

\section{CITATION:}

Kuroi, Kunisato ...[et al]. Time-resolved fluctuation during the photochemical reaction of a photoreceptor protein: Phototropin1LOV2-linker. Physical Chemistry Chemical Physics 2016, 18: 6228-6238

\section{ISSUE DATE:}

2016-01-27

URL:

http://hdl.handle.net/2433/218348

\section{RIGHT:}

This is the accepted version of the following article, which has been published in final form at http://dx.doi.org/10.1039/c5cp07472j.; This is not the published version. Please cite only the published version.; この論文は出版社版でありません。引用の際には出版社 版をご確認ご利用ください。 


\section{Time-Resolved Fluctuation during the Photochemical Reaction of a Photoreceptor Protein: Phototropin1LOV2-Linker}

Kunisato Kuroi, ${ }^{\dagger}$ Francielle Sato, ${ }^{\dagger, \#}$ Yusuke Nakasone, ${ }^{\dagger}$ Kazunori Zikihara, ${ }^{\S}$ Satoru Tokutomi, ${ }^{\S}$ and Masahide Terazima ${ }^{* \dagger}$

${ }^{\dagger}$ Department of Chemistry, Graduate School of Science, Kyoto University, Kyoto 606-8502, Japan ${ }^{\S}$ Department of Biological Science, Graduate School of Science, Osaka Prefecture University, Sakai, Osaka 599-8531, Japan

\#Present address: Departamento de Física, Universidade Estadual de Maringá, Av. Colombo 5790, CEP: 87020-900, Maringá-PR, Brasil

* corresponding author:

Masahide Terazima

e-mail: mterazima@,kuchem.kyoto-u.ac.jp

tel/FAX: +81-75-753-4026 
ABSTRACT: Although the relationship between structural fluctuations and reactions is important for elucidating reaction mechanisms, experimental data describing such fluctuations of reaction intermediates are sparse. In order to investigate structural fluctuations during a protein reaction, the compressibilities of intermediate species after photoexcitation of phot1LOV2-linker, which is a typical LOV domain protein with the C-terminal linker including the J- $\alpha$ helix and used recently for optogenetics, were measured in the time-domain by the transient grating and transient lens methods with a high pressure optical cell. The yield of covalent bond formation between the chromophore and a Cys residue (S state formation) relative to that at $0.1 \mathrm{MPa}$ decreased very slightly with increasing pressure. The fraction of the reactive species that yields the $\mathrm{T}$ state (linker-unfolded state) decreased almost proportionally with pressure $(0.1-200 \mathrm{MPa})$ to about $65 \%$. Interestingly, the volume change associated with the reaction was much more pressure sensitive. Combined these data, the compressibility changes for the short lived intermediate ( $\mathrm{S}$ state) and the final product ( $\mathrm{T}$ state) formation were determined. The compressibility of the S state was found to increase compared with the dark (D) state, and the compressibility decreased during the transition from the S state to the T state. The compressibility change is discussed in terms of cavities inside the protein. By comparing the crystal structures of phot1LOV2linker at dark and light states, we concluded that the cavity volumes between the LOV domain and the linker domain increase in the S state, which explains the enhanced compressibility. 


\section{INTRODUCTION}

Understanding the photochemical reaction of the LOV (LOV= light, oxygen, and voltage sensor) domain has become increasingly more important because this domain can be used in various applications, including the recent use as a tool for optogenetics. ${ }^{1,2}$ Thus, detailing the molecular mechanism of this reaction is an important issue in physical chemistry and in potential LOV domain-based applications, e.g., to control such a reaction, the driving force of the reaction must be understood in detail. In the LOV domain, a chromophore, flavin mononucleotide (FMN), is photoexcited upon blue light irradiation and the initial reaction, the adduct formation between FMN and an adjacent cysteine residue of the LOV domain, takes place. ${ }^{3}$ This reaction leads to subsequent reactions, such as changes in the intermolecular interaction (the dimerization/dissociation reaction of the LOV domains) and/or the inter-domain interaction (dissociation of the linker domain, as described below). Naturally, a conformational change to the LOV domain could be considered to be a driving force that facilitates subsequent reactions. However, according to theoretical and experimental studies, the average structure of the LOV domain does not undergo sizeable conformational changes. ${ }^{4-12}$ Recently, the importance of structural fluctuations has been revealed for reactions of protein systems. ${ }^{13-21}$ In general, proteins are flexible systems and structures should fluctuate at room temperature in aqueous solution owing to thermal energy. ${ }^{13}$ These fluctuations are hypothesized to be important for the biological functions of proteins, e.g., signal transport ${ }^{17}$ and enzyme activity. ${ }^{18,19}$ However, experimental data to link protein fluctuations and reactivity are limited. To achieve such a link, data must be collected on a time trace of the fluctuation along the reaction coordinate. Unfortunately, experimental detection of such conformation fluctuations is not trivial, in particular, in the time-domain.

For elucidating the importance of protein fluctuations, the thermodynamic properties should be important. Although measurements of thermodynamic properties of short-lived intermediates are very difficult, we have developed a method of the time-resolved measurement during reactions based on the 
time-resolved transient grating (TG) method. ${ }^{22-26}$ In particular, the partial molar isothermal compressibility $\left.\left(\bar{K}_{T}=-(\partial \bar{V} / \partial P)_{T}\right)\right)$ is essential for elucidating the fluctuation, ${ }^{27-30}$ because this property is directly linked to the mean-square fluctuations of the protein partial molar volume by $\left\langle(\bar{V}-\langle\bar{V}\rangle)^{2}\right\rangle \equiv\left\langle\delta \bar{V}^{2}\right\rangle=k_{B} T \bar{K}_{T}{ }^{31}$ (Here, $<\mathrm{X}>$ means the average value of a quantity X.) By developing a high pressure cell for the TG measurement, ${ }^{32}$ we succeeded in measuring the compressibility change during the photodissociation reaction of a photosensor protein with a BLUF (sensors of blue light $\underline{u}$ sing FAD) domain, TePixD. ${ }^{33}$ In the present paper, we measured the partial molar isothermal compressibility change of intermediates of a typical LOV domain protein, the phot1LOV2-linker of photropin (phot) of Arabidopsis thaliana, to show the fluctuations of reaction intermediates.

Phototropins are blue-light photoreceptor proteins in plants and green microalgae, and are involved in phototropism, chloroplast movement and stomata opening in plants. ${ }^{34}$ Higher plants such as A. thaliana have two isoforms of phototropin (phot): phot1 and phot2. These phots comprise N-terminal blue-light sensing domains, LOV1 and LOV2, a serine-threonine kinase domain in the C-terminal region and a linker domain that includes the J- $\alpha$ helix and connects the LOV2 and the kinase domain. For blue-light absorption, the LOV1 and LOV2 domains bind a FMN molecule non-covalently. Upon blue light irradiation of the dark state (the D state), the photo-cyclic reaction occurs; photoexcited FMN undergoes FMN-C4a-cysteinyl adduct formation with an adjacent cysteine residue of the LOV domain (the S state). ${ }^{3,}$ ${ }^{34}$ After the S state formation, the linker helix is unfolded with a time constant of $1 \mathrm{~ms}$ (the T state). ${ }^{35-38}$ Reactive and non-reactive species have been reported to exist in solution, which was concluded from the temperature dependence of the TG signal. ${ }^{39}$ Here, the non-reactive species implies the species that does not yield the $\mathrm{T}$ state after $\mathrm{S}$ state formation. The photoproduct is recovered back to the $\mathrm{D}$-state with a time constant of about $1 \mathrm{~min}$ at room temperature and ambient pressure. ${ }^{38,40,41}$ The reaction scheme we used for the present study is illustrated in Fig. 1. 
Fig. 1

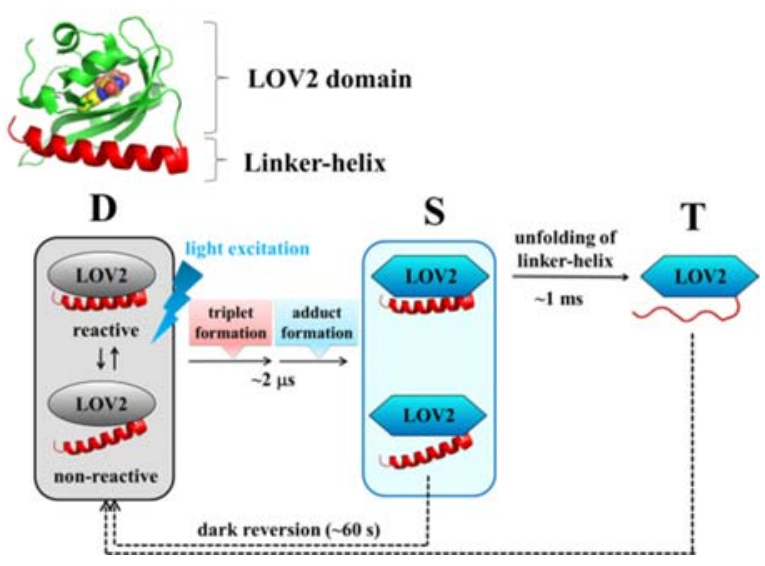

Figure 1. (Upper) Crystal structure of the phot1LOV2-linker(2V1A). LOV2 domain and the linker-helix (J $\alpha$-helix) are depicted by green and red. (Lower) Reaction scheme of the phot1LOV2-linker. Round and hexagonal shapes represent the different states of the LOV2 domain. The S and T states cannot be distinguished by a UV/vis absorption method. There are reactive and nonreactive species in the S state. The reactive species transform to the $\mathrm{T}$ state, whereas the nonreactive species do not.

In this study, we investigated the compressibility change during the reaction of the phot1LOV2linker by the following procedure. First, the pressure dependence of the reaction yields of the S state formation was measured by the transient absorption method. In addition, the pressure dependence of the yield of the $\mathrm{T}$ state formation from the $\mathrm{S}$ state was detected by the diffusion signal of the TG component in Section 3.1. Second, using these data on the pressure dependence of the reaction yield, the compressibility change for the $\mathrm{S} \rightarrow \mathrm{T}$ transition was determined from the pressure dependence of the volume changes measured by the TG and transient lens ( $\operatorname{TrL}$ ) methods in Section 3.2. The compressibility difference between the initial $\mathrm{D}$ state and the final state, which is a mixture of the $\mathrm{S}$ and $\mathrm{T}$ states was also determined by the TrL method, and the contributions from the $\mathrm{S}$ and $\mathrm{T}$ states were separated by a detailed 
analysis in Section 3.3. Finally, the results are discussed with respect to the cavities inside the protein.

\section{EXPERIMENTAL SECTION}

Preparation of Sample. The preparation method of the Arabidopsis phot1-LOV2-linker (449E$661 \mathrm{~K})$ polypeptide was reported previously. ${ }^{41,42}$ The polypeptide was expressed with the pGEX6P-1 vector in an Escherichia coli strain (JM109). The GST-tagged protein was purified by a GSTrap column (GE Healthcare) and GST tags were removed by a PreScission Protease digestion. The cleaved polypeptides were further purified by gel chromatography with Sephacryl S-100 HR (GE Healthcare) and eluted with phosphate-buffered saline containing $140 \mathrm{mM} \mathrm{NaCl}, 10 \mathrm{mM} \mathrm{Na}_{2} \mathrm{HPO}_{4}, 2.7 \mathrm{mM} \mathrm{KCl}$ and $1.8 \mathrm{mM} \mathrm{KH}_{2} \mathrm{PO}_{4}(\mathrm{pH}$ 7.5). The sample was dissolved in the same buffer solution. The purified polypeptide was concentrated by ultrafiltration and then used for the measurements. The concentration of the sample was determined by the absorbance at $447 \mathrm{~nm}$ using the extinction coefficient of 14300 $\mathrm{M}^{-1} \mathrm{~cm}^{-1}$. The concentration of the samples was usually $\sim 500 \mu \mathrm{M}$.

High-Pressure Equipment. Details of the high-pressure apparatus used in this study have been described elsewhere. ${ }^{32}$ The pressure resistance of this cell was up to $500 \mathrm{MPa}$. High pressure was generated by pumping water with a compact hand pump (Syn Corporation TP-501, Kyoto, Japan). The sample temperature was controlled by circulating temperature-controlled water through a hole drilled within the cell. For absorption, TG and TrL measurements under high pressure, the sample solution was encapsulated in the high pressure inner cell and incorporated into the high-pressure apparatus. This high pressure cell achieves complete reproducibility of a signal on applying high pressure and a sample replacement operation. The pressure was reset to $0.1 \mathrm{MPa}$ after every compression (high pressure experiment) to check the recovery of the signal. The signals were confirmed to be completely reversible.

TG and TrL Measurements. For TG and TrL measurements, a $\mathrm{XeCl}$ excimer laser-pumped dye laser beam (Lambda Physik CompexPro102; $\lambda=308 \mathrm{~nm}$, Lumonics Hyper Dye $300 ; \lambda=462 \mathrm{~nm}$ ) was 
used for the excitation laser. The signal was detected by a photomultiplier tube (R1477; Hamamatsu). The signal was fed into a digital oscilloscope (TDS-7104; Tektronix) and averaged. The laser beams were focused slightly on the sample solution inside the high-pressure apparatus, the repetition rate of excitation was usually $0.015 \mathrm{~Hz}$ and the laser power for excitation was usually set to be weak enough $(<20 \mu \mathrm{J}$ puls $^{-1}$ ) to not excite the photoexcited protein twice by the laser pulse. It was confirmed that the TG signal intensity did not change further by decreasing the repetition rate to less than $0.015 \mathrm{~Hz}$ even at high-pressure. The experimental setup for the TG measurement was similar to that reported before. ${ }^{32,33}$ Briefly, in the TG method, two laser pulses are introduced into the sample solution. A CW diode laser (Crysta Laser, $835 \mathrm{~nm}$ ) was used as a probe light source. The grating wavenumber, $q$ value, at each experimental setup was determined from the decay rate of the thermal grating signal of the calorimetric reference (aqueous solution of bromocresol purple). The intensity ( $\left.I_{\mathrm{TG}}\right)$ is proportional to the square of the generated refractive index change $(\delta n)$ arising from the temperature change (thermal grating, $\delta n_{\text {th }}$ ), volume change $\left(\delta n_{\mathrm{v}}\right)$ and absorption change $\left(\delta n_{\mathrm{pop}}\right) .{ }^{25,43,44}$ The origins of the TG signal are described in more detail in the Supporting Information (SI-1(a)).

The experimental setup for the TrL measurements was reported previously. ${ }^{45}, 46$ The $\mathrm{XeCl}$ excimer laser-pumped dye laser beam (462 nm) was used for the excitation, and a He-Ne laser (632 nm) was used for the probe beams. The excitation beam was focused slightly by a lens with a focal length of $20-40 \mathrm{~cm}$. The probe beam was introduced into the sample collinearly with the pump beam. After passing through the sample, the probe beam was expanded using a concave lens, and the $\operatorname{TrL}$ signal was detected as a light intensity change at the beam center using a pinhole. The intensity of the $\operatorname{TrL}$ signal at a time $t\left(I_{\operatorname{TrL}}(\mathrm{t})\right)$ was defined as the ratio of the probe light intensity change at time $t$ to the initial intensity at $t=0$ (just before the excitation) (SI-1(b)). The intensity of the TrL signal is proportional to the refractive index change induced by light excitation. ${ }^{46}$ The origins of the TrL signal are described in more detail in the Supporting Information (SI-1(b)). 
Absorption Measurements. UV-Vis absorption spectra at various pressures were measured by a UV-Vis spectrometer (Shimadzu UV-2550) with the high-pressure cell. The temporal profile of the transient absorption signal was monitored at $483 \mathrm{~nm}$ after photo-excitation by the same pump pulse used for the TG measurement.

\section{RESULTS}

3.1 Pressure Dependent Reaction Yield. If the reaction yield does not depend on the pressure, the compressibility can be determined simply by measuring the volume changes after photoexcitation with a constant excitation light intensity at various pressures. However, since proteins are generally flexible, the protein reaction yield could be pressure dependent. ${ }^{47,}{ }^{48}$ Thus, we first examined the pressure dependence of the reaction yield of the phot1LOV2-linker. The pressure effects on the UV-Vis absorption spectra in the dark state were found to be negligible after the correction of the increase in the density of the solution (i.e., concentration) (Fig. S-1). This fact ensures that the conformation, at least around the chromophore, does not change with pressure over the range of $0.1-400 \mathrm{MPa}$.

The pressure dependence of the reaction yield of the $\mathrm{S}$ state formation was measured by the transient absorption signal. Fig. 2(a) depicts the time profiles of the absorption changes after the photoexcitation of the phot1LOV2-linker at various pressures. The amplitudes, which represent the yield of the S (and T) state formation, and the lifetimes, are plotted in Fig. 2(b) and (c), respectively. 
Fig. 2

(a)

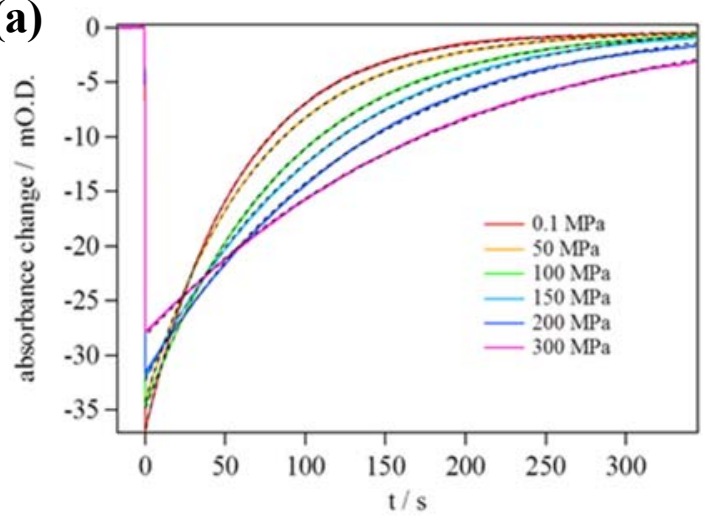

(c)

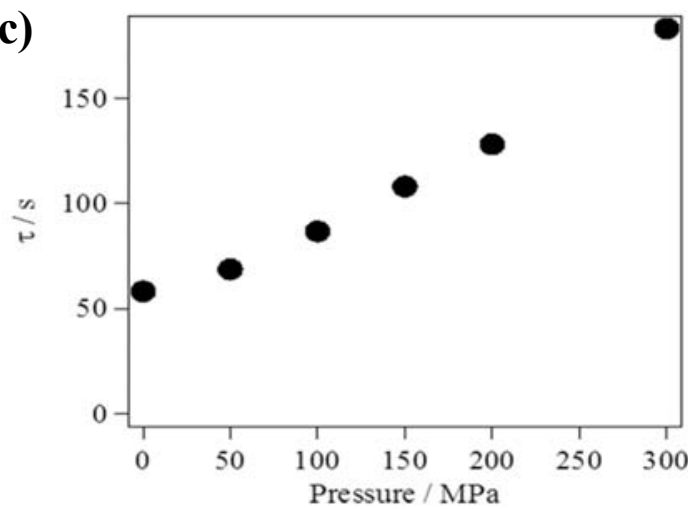

(b)

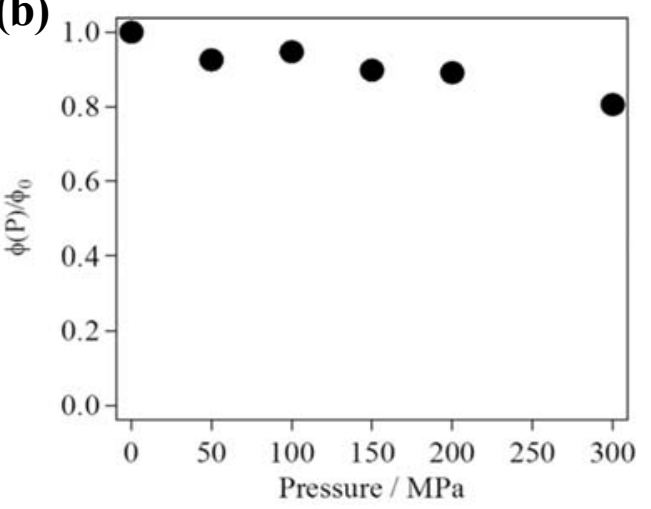

Figure 2. (a) Temporal profiles of the absorption change of the phot1LOV2-linker after excitation at 462 $\mathrm{nm}$ recorded at $483 \mathrm{~nm}$. Pressures are shown in the legend in the figure. The best-fit curves by a singleexponential function are shown by broken lines. (b) Pressure dependence of the relative quantum yield $(\phi(\mathrm{P}))$ of the photoreaction of the phot1LOV2-linker, which was obtained from the amplitudes of the time profiles of (a) normalized by the quantum yield at $0.1 \mathrm{MPa}(\phi 0)$. (c) Pressure dependence of the time constant of the dark recovery.

The rate constant of the recovery decreased with increasing pressure. The yield of the $\mathrm{S}$ state formation at a pressure $\mathrm{P}(\phi(\mathrm{P}))$ relative to that at $0.1 \mathrm{MPa}\left(\phi_{0}\right)$ decreased slightly with increasing pressure, although the dependence was minor. To determine the compressibility change described later, this 
apparent pressure dependence should be taken into account. Hereafter, observed quantities (e.g., volume and compressibility changes) were corrected by the pressure dependence of this yield $\left(\phi(\mathrm{P}) / \phi_{0}\right)$ and the density change; that is, the quantities below are corrected for the density change and normalized by the S state formation.

The reaction yield of the T state formation was measured by the diffusion signal of the TG method. The time-evolution of the TG signal after photoexcitation of the phot1LOV2-linker at $0.1 \mathrm{MPa}$ has been reported previously. ${ }^{36}$ A typical TG signal over a wide time range at ambient pressure is shown in Fig. S2. The TG signal consists of five components: the adduct formation process (occurs over a few $\mu$ s), the thermal grating component (decay rate constant $D_{\text {th }} \mathrm{q}^{2}$ ( $D_{\text {th }}$ : thermal diffusivity)), a volume contraction process associated with the transition from the $\mathrm{S}$ state to the $\mathrm{T}$ state with a time constant of $\sim 1 \mathrm{~ms}$ (not clearly seen in this signal, vide infra), and a peak of the molecular diffusion signal, which represents the diffusion of the T and D species. By taking the sum of these contributions, the signal can be reproduced well.

After the complete unfolding reaction of the linker part, the molecular diffusion signal represents the diffusion of the reactant (D species) and the product (T species) (SI-1). Therefore, the signal should be expressed by:

$$
I_{\mathrm{TG}}(\mathrm{t})=\alpha\left\{\delta n_{\mathrm{T}} \exp \left(-D_{\mathrm{T}} q^{2} t\right)-\delta n_{\mathrm{D}} \exp \left(-D_{\mathrm{D}} q^{2} t\right)\right\}^{2}
$$

where $\alpha$ is a constant representing the sensitivity of the detection system, $D_{\mathrm{T}}$ and $D_{\mathrm{D}}$ are the diffusion coefficients of the T and D species, respectively. Furthermore, $\delta n_{\mathrm{D}}$ and $\delta n_{\mathrm{T}}$ are the refractive index changes of the $\mathrm{D}$ state and the $\mathrm{T}$ state, respectively. It should be mentioned that the diffusion coefficient of the S state (without unfolding of the linker) does not change from that of the D state, and hence this contribution can be neglected in the diffusion signal. ${ }^{5,22}$ Hence, the diffusion signal intensity represents 
the yield of the $\mathrm{T}$ state formation after photoexcitation.

Fig. 3(a) depicts the diffusion signals of the phot1LOV2-linker at various pressures.

Fig. 3
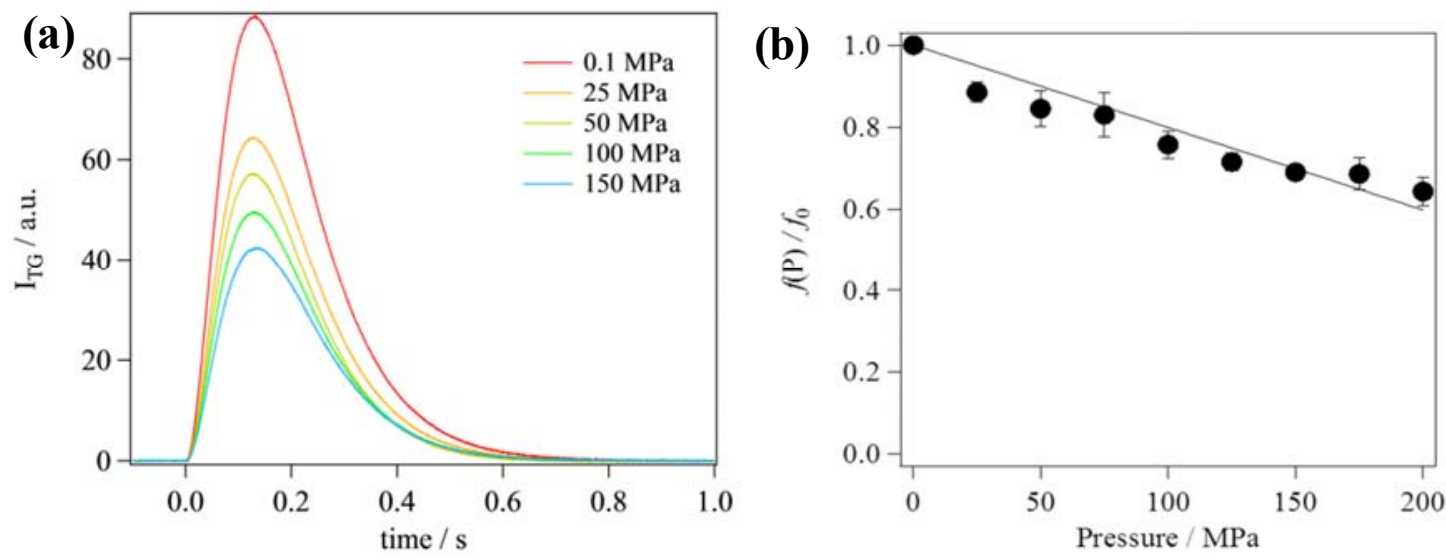

Figure 3. (a) Pressure dependence of the diffusion signal. Pressures are indicated by the legend in the figure. (b) Pressure dependence of the yield of the T species (reactive species) formation relative to $P=$ $0.1 \mathrm{MPa}\left(f_{0}\right)$. The best fitted line by a linear function of $P$ is shown by the solid line.

It is important to note that the peak intensity of the diffusion signal decreased with increasing pressure. Since the temporal profile did not depend on the pressure within a range of $0.1-150 \mathrm{MPa}$ (Fig. S-3), the observed intensity decrease is not due to a possible pressure dependent $D$ change by high pressure, but because of a decrease in the reaction yield. The pressure dependence is much larger than that of the $\mathrm{S}$ state formation described above. Hence, this pressure dependence must come from the pressure dependent reaction yield after S state formation. Since there are reactive species that can lead to the $\mathrm{T}$ state formation and non-reactive species that do not form the $\mathrm{T}$ state in solution, ${ }^{39}$ the present result indicates that the fraction of reactive and non-reactive species is pressure dependent. The pressure 
dependence of the fraction of the reactive species $f(\mathrm{P})$ normalized by $f(0.1 \mathrm{MPa})\left(\equiv f_{0}\right)$ is plotted against $P$ in Fig. 3(b). The figure shows that $f(\mathrm{P})$ decreases almost proportionally to pressure over the pressure range studied. By fitting the pressure dependence, we obtained a pressure dependence of $f(\mathrm{P})$ to be $f_{0}\left(1-2.0 \times 10^{-3} \mathrm{P}\right)$. This pressure dependent fraction was used for calculating the thermodynamic properties of the $\mathrm{T}$ state below.

The fraction at ambient pressure $\left(f_{0}\right)$ was estimated from the temperature dependence of the diffusion signal of the phot1LOV2-linker. The non-reactive form was suggested previously based on an observation that the diffusion signal intensity increased with decreasing temperature. ${ }^{39}$ However, in this buffer solution, we found that the temperature dependence of the diffusion signal was minor and the diffusion peak intensity did not increase below $283 \mathrm{~K}$ (Fig. S-4). This result indicates that most of the S species is transformed to the $\mathrm{T}$ state at ambient pressure; i.e., $f_{0} \sim 1$. However, we corrected the quantities below by a factor of $f(\mathrm{P})=f_{0}\left(1-2.0 \times 10^{-3} \mathrm{P}\right)$. One may recalculate the quantities easily even if $f_{0}$ is less than unity.

3.2 Compressibility Change During the $\mathbf{S} \rightarrow \mathbf{T}$ Process. For measuring the volume change associated with the unfolding process of the linker ( $\mathrm{S} \rightarrow \mathrm{T}$ process), we used the TG and TrL methods. When we excited the calorimetric reference sample, which releases all the energy of the photoexcited state as heat, the TrL signal (thermal lens signal in this case) decayed within $300 \mathrm{~ms}$ (vide infra). Fig. 4(a) shows the TrL signals over a range of $0-3.5 \mathrm{~ms}$ after photoexcitation of the phot1LOV2-linker and the calorimetric reference sample at $0.1 \mathrm{MPa}$. 
Fig. 4
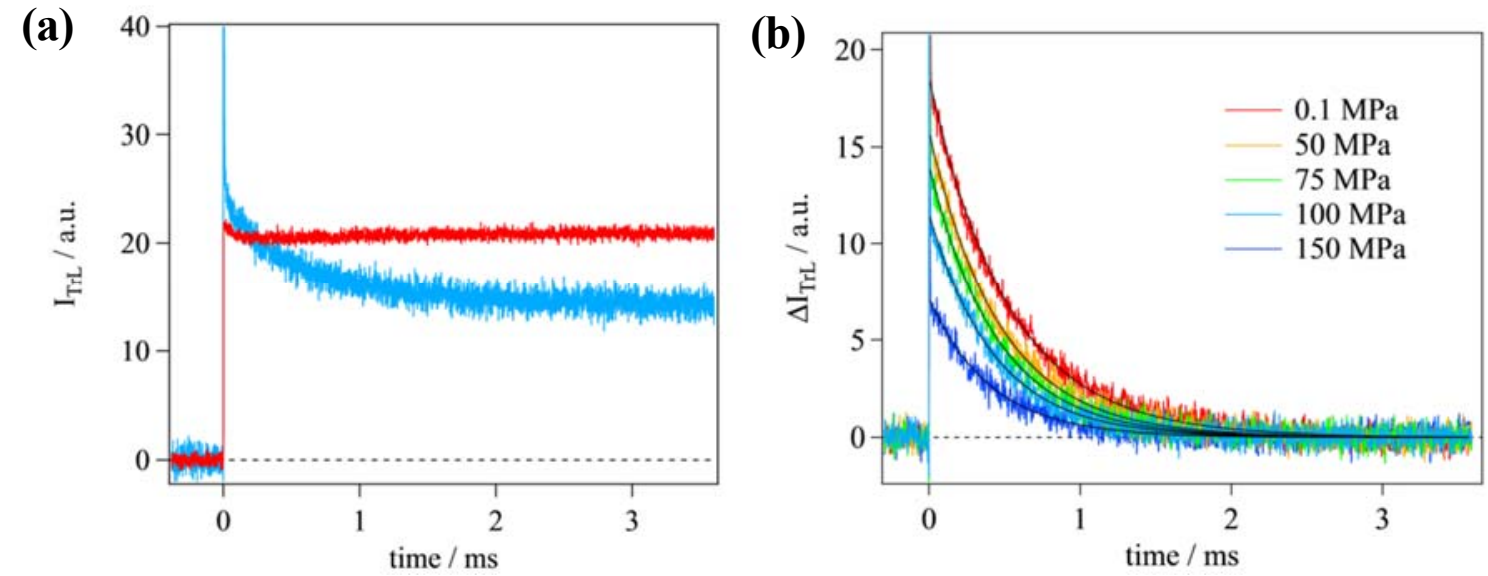

Figure 4. (a) Typical examples of the TrL signals of the the phot1LOV2-linker (blue line) and of the calorimetric reference sample (thermal lens signal: red line) at ambient pressure. (b) Temporal profile of the TrL signals representing the reaction dynamics from the $\mathrm{S}$ state to the $\mathrm{T}$ state (linker unfolded state) at various pressures. Pressures are indicated by the legend in the figure.

In this time range, the thermal lens signal intensity is almost constant. The unfolding process of the linker was detected as the decay of the TrL signal on this thermal lens signal. ${ }^{36}$ The $\operatorname{TrL}$ signal $(\operatorname{IrL}(\mathrm{t}))$ in this time range should be expressed by:

$$
I_{\mathrm{TrL}}(\mathrm{t})=\beta\left\{\delta n_{\mathrm{th}}^{\prime}(t)+\left(\delta n_{\mathrm{T}}-\delta n_{\mathrm{S}}\right) \exp \left(-k_{\mathrm{ST}} t\right)+C\right\}
$$

where $\beta$ is a constant representing the instrument sensitivity, $\delta n^{\prime}$ th $(t)$ is the temporal profile of the thermal lens signal, which was determined from the TrL signal of the calorimetric reference sample, $k_{\mathrm{ST}}$ is the rate constant of the unfolding process, and $\left(\delta n_{\mathrm{T}}-\delta n_{\mathrm{S}}\right)$ is the refractive index difference between the $\mathrm{S}$ and $\mathrm{T}$ states. Furthermore, the protein diffusion process of the $\mathrm{T}$ and $\mathrm{D}$ states occurs over a much longer time 
range (i.e., longer than $\sim 10 \mathrm{~s}$ ) in the TrL experiment (vide infra), so that this contribution is expressed by a constant $\mathrm{C}$ in Eq. (2). We fitted the observed signal by Eq. (2). In order to clearly show the contribution of the unfolding process in the $\operatorname{TrL}$ signal, $\delta n^{\prime}$ th $(\mathrm{t})$ and $C$ contributions, both of which are timeindependent in the present time range, were subtracted from the signal and the $\operatorname{TrL}$ signals associated with the unfolding reaction at various pressures (i.e., $\left.\beta\left(\delta n_{\mathrm{T}}-\delta n \mathrm{~s}\right) \exp (-k \mathrm{sTt})\right)$ are shown in Fig. 4(b). Clearly, the amplitude that represents the unfolding process decreased with increasing pressure. We determined the rate constant of the unfolding reaction $k_{\mathrm{ST}}$ at various pressures by fitting these signals with a single exponential function (Fig. 5(a)).

Fig. 5
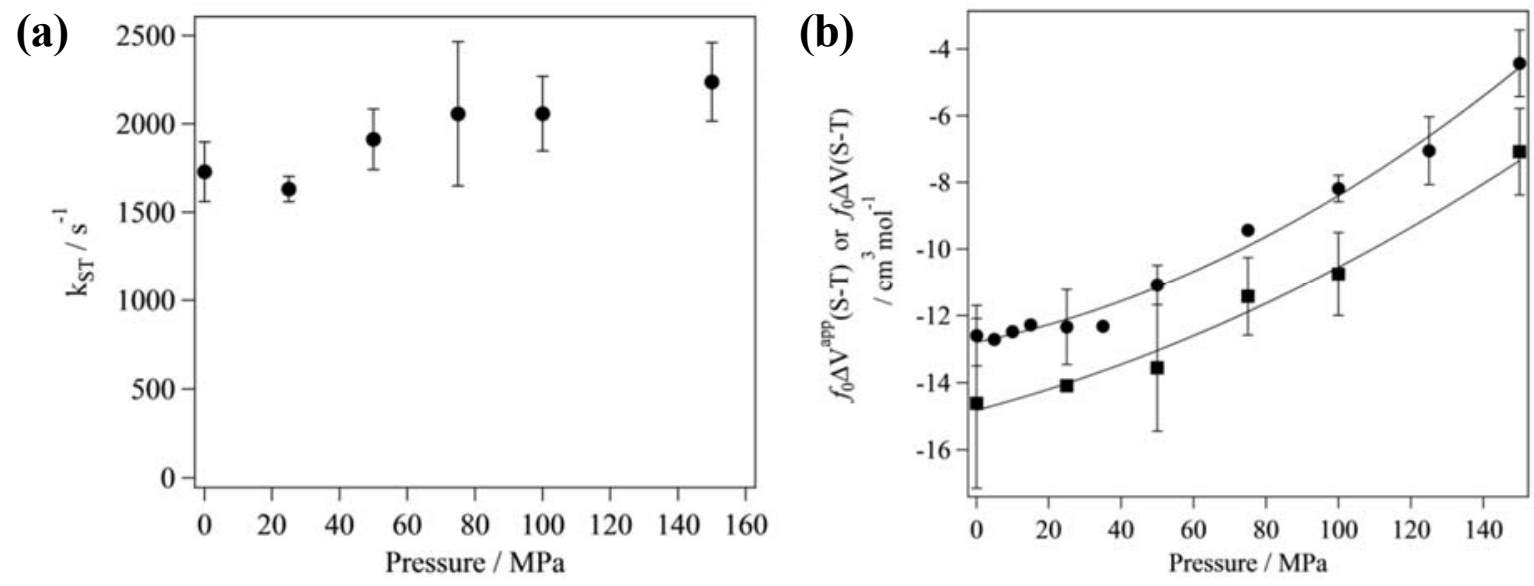

Figure 5. (a) Pressure dependence of the rate constant of the reaction from the $\mathrm{S}$ state to the $\mathrm{T}$ state determined from the signals in Fig. 4. (b) Pressure dependence of the apparent volume change ( $f_{0} \Delta V^{\text {app }}(\mathrm{S}$ $\mathrm{T})$ ) determined from the $\operatorname{TrL}$ signal (squares) and the volume change $\left(f_{0} \Delta V(\mathrm{~S}-\mathrm{T})\right)$ determined from the TG signal (circles). The best fitted line by a quadratic function of $P$ is shown by the solid lines.

The rate constant of the reaction increased slightly with increasing pressure, but not significantly. 
This observation indicates that the activation volume is negative but small for this reaction.

There are two contributions in the origin of the amplitude $\left(\delta n_{\mathrm{T}}-\delta n_{\mathrm{s}}\right)$. Under the $\operatorname{TrL}$ experimental condition, the decay of the thermal lens signal due to the thermal diffusion (an order of $\sim 100$ ms (vide infra)) is much slower than the time constant of the unfolding reaction $(\sim 1 \mathrm{~ms})$. Hence, the thermal contribution accumulates over this time range, such that the amplitude $\left(\delta n_{\mathrm{T}}-\delta n_{\mathrm{S}}\right)$ must reflect the volume change $(\Delta V(\mathrm{~S}-\mathrm{T}))$ as well as the enthalpy change $(\Delta H(\mathrm{~S}-\mathrm{T}))$ for this reaction. Quantitatively, as shown in Eq. (S-13), this amplitude divided by the thermal lens signal intensity of a calorimetric reference sample is related by the factor $f_{0}\left\{\Delta V(\mathrm{~S}-\mathrm{T})-\left(\alpha_{\mathrm{th}} W / \rho C_{\mathrm{p}}\right) \Delta H(\mathrm{~S}-\mathrm{T})\right\}$. Here, $\alpha_{\mathrm{th}}$ represents the thermal expansion coefficient of the solvent, $W$ is the molecular weight $\left(\mathrm{g} \mathrm{mol}^{-1}\right), \rho$ is the density $\left(\mathrm{g} \mathrm{L}^{-1}\right)$ and $C_{\mathrm{p}}$ is the heat capacity $\left(\mathrm{J} \mathrm{K}^{-1} \mathrm{~mol}^{-1}\right)$. Since the dimension of this quantity is the volume $\left(\mathrm{mL} \mathrm{mol}^{-1}\right)$, we refer to this quantity as the apparent volume change $\left(\Delta V^{\mathrm{app}}(\mathrm{S}-\mathrm{T})\right)$. We calculated this value at various pressures, and plotted the results in Fig. 5(b).

This plot was well reproduced by the quadratic function of the pressure $(P)$ :

$$
f_{0} \Delta V^{\mathrm{app}}(\mathrm{S}-\mathrm{T})=K_{0}+K_{1}(\mathrm{P}-0.1)+K_{2}(\mathrm{P}-0.1)^{2}
$$

From this fitting, we obtained $K_{0}=-15 \pm 0.3 \mathrm{~cm}^{3} \mathrm{~mol}^{-1}, K_{1}=(2.9 \pm 0.8) \times 10^{-2} \mathrm{~cm}^{3} \mathrm{~mol}^{-1} \mathrm{MPa}^{-1}$ and $K_{2}=-(1.4 \pm 0.4) \times 10^{-4} \mathrm{~cm}^{3} \mathrm{~mol}^{-1} \mathrm{MPa}^{-2}$. If the pressure dependence of the enthalpy contribution is negligible (i.e., $(\partial \Delta H(\mathrm{~S}-\mathrm{T}) / \partial P) \sim 0)$, the compressibility change at ambient pressure can be determined from the pressure derivative of Eq. (3) with $P=0.1 \mathrm{MPa}$ to be $-K_{1}=-2.9 \times 10^{-2} \mathrm{~cm}^{3} \mathrm{~mol}^{-1} \mathrm{MPa}^{-1}$. In order to examine if the pressure dependence of $\Delta H$ is negligible, we used the TG method as follows.

For the reaction of the phot1LOV2-linker, the TG signal consists of the thermal grating, the volume change, and the protein diffusion processes. Since the thermal grating and the diffusion signals are very 
strong compared with the signal from a volume change, the volume grating signal is easily masked by these strong components and it is difficult to determine the intensity. However, the rate constant of the thermal diffusion $\left(D_{\mathrm{th}} q^{2}\right)$ and that of the protein diffusion $\left(D_{\mathrm{D}} q^{2}\right.$ and $\left.D_{\mathrm{T}} q^{2}\right)$ depend on the $q^{2}$-value, while the rate of the volume change process is independent of the $q^{2}$-value. Hence, by choosing appropriate $q^{2}$ values, we can reduce the disturbance that overlaps with the volume change signal. For this purpose, we used $q^{2}=2.8 \times 10^{11} \mathrm{~m}^{-2}$ and the TG signal in a time range of $50 \mathrm{~ns}-3 \mathrm{~ms}$ is shown in Fig. 6 .

Fig. 6

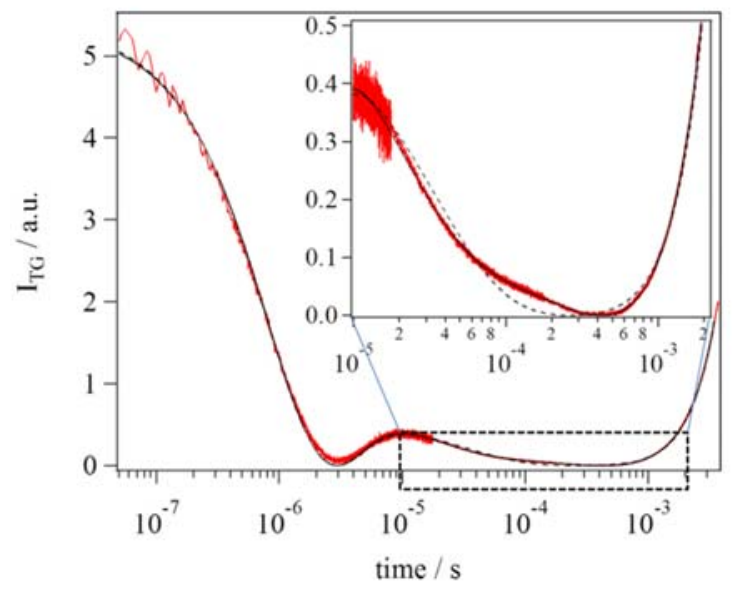

Figure 6. TG signal at $q^{2}=2.8 \times 10^{11} \mathrm{~m}^{-2}$ at ambient pressure for determining the volume change associated with the $\mathrm{S}$ state to $\mathrm{T}$ state transition. The signal decaying with a time constant of $30 \mu$ s is the thermal grating component, the rising signal after $1 \mathrm{~ms}$ is part of the rise of the diffusion signal. The best fitted signal without a volume contribution is shown by the broken line, which does not reproduce the signal. The best fitted signal taking into account a volume change (solid line) reproduced the signal well.

The temporal profile of the TG signal under this condition should be expressed by: 


$$
\begin{aligned}
I_{\mathrm{TG}}(\mathrm{t}) & =\alpha\left\{\delta n_{\mathrm{ad}} \exp \left(-k_{\mathrm{ad}} t\right)+\delta n_{\mathrm{th}} \exp \left(-D_{\mathrm{th}} q^{2} t\right)+\left(\delta n_{\mathrm{T}}-\delta n_{\mathrm{S}}\right) \exp \left(-k_{\mathrm{ST}} t\right)\right. \\
& \left.+\delta n_{\mathrm{T}} \exp \left(-D_{\mathrm{T}} q^{2} t\right)-\delta n_{\mathrm{D}} \exp \left(-D_{\mathrm{D}} q^{2} t\right)\right\}^{2}
\end{aligned}
$$

where $\delta n_{\mathrm{ad}}$ and $k_{\mathrm{ad}}$ are the refractive index change and the rate constant of the adduct formation, respectively. In order to determine the amplitude of the volume change without ambiguity, we fitted the signal over the time range of $50 \mathrm{~ns}-3 \mathrm{~ms}$ by using an approximation of:

$$
\delta n_{\mathrm{T}} \exp \left(-D_{\mathrm{T}} q^{2} t\right)-\delta n_{\mathrm{D}} \exp \left(-D_{\mathrm{D}} q^{2} t\right) \sim \mathrm{A}+\mathrm{Bt}
$$

where $A$ and $B$ are adjustable constants. This approximation is reasonable, because the rate constants in the exponential functions (an order of $300 \mathrm{~ms}$ ) are smaller than the present time range $(0<\mathrm{t}<2 \mathrm{~ms})$, so the exponential function can be expanded as $\delta n_{\mathrm{T}} \exp \left(-D_{\mathrm{T}} q^{2} t\right) \sim \delta n_{\mathrm{T}}\left(1-D_{\mathrm{T}} q^{2} t\right)$ and $\delta n_{\mathrm{D}} \exp \left(-D_{\mathrm{D}} q^{2} t\right) \sim$ $\delta n_{\mathrm{D}}\left(1-D{ }_{\mathrm{D}} q^{2} t\right)$. Hence, the constants $A$ and $B$ are given by $A=\delta n_{\mathrm{T}}-\delta n_{\mathrm{D}}$ and $B=-\delta n_{\mathrm{T}} D q_{\mathrm{T}}^{2}+\delta n_{\mathrm{D}} D{ }_{\mathrm{D}} q^{2}$. These are adjustable constants in the fitting. If we tried to fit the signal without the volume grating contribution, i.e., $\left(\delta n_{\mathrm{T}}-\delta n \mathrm{~s}\right)=0$, we could not reproduce the signal (Fig. 6, inset); hence, it is apparent that the volume grating component contributes to the signal. Furthermore, the value of $k \mathrm{st}$ in Eq. (4) was fixed, as determined at each pressure from the TrL signal. Using these approximations and restrictions, we determined ( $\left.\delta n_{\mathrm{T}}-\delta n_{\mathrm{S}}\right)$ without any ambiguity.

The difference between $\left(\delta n_{\mathrm{T}}-\delta n \mathrm{~s}\right)$ from the TG signal and that from the previous TrL signal comes from the different rate of the thermal diffusion. Since the decay of the thermal grating signal is much faster than the rate constant of $k_{\mathrm{ST}}$ under the present TG experimental condition, the thermal contribution ( $\Delta H$ term) dissipated rapidly and can be ignored. Hence the volume grating signal comes only from the volume change of the reactive form $f(\mathrm{P}) \Delta V(\mathrm{~S}-\mathrm{T})$. The volume change $f_{0} \Delta V(\mathrm{~S}-\mathrm{T})$ was calculated from 
$\left(\delta n_{\mathrm{T}}-\delta n_{\mathrm{S}}\right)$ and the amplitude of the TG signal from the calorimetric reference sample $\left(\delta n_{\mathrm{th}}^{\mathrm{ref}}\right)$ by a method described in (SI-6), and plotted against $P$ in Fig. 5(b). The values from the TG signal are different from those from the TrL signal. The difference must come from the enthalpy contribution. However, it is interesting to note that the pressure dependences of the $\left(\delta n_{\mathrm{T}}-\delta n_{\mathrm{S}}\right) \mathrm{vs}$. $P$ plot from the TG signal and that from the TrL signal are similar (Fig. 5(b)). This agreement indicates that the enthalpy contribution $\Delta H(\mathrm{~S}-$ $\mathrm{T})$ in $\left(\delta n_{\mathrm{T}}-\delta n_{\mathrm{S}}\right)$ is not pressure dependent.

The pressure dependence of this plot was well reproduced by the quadratic function of $P$ (Eq. (3)). The slope of the plot at $0.1 \mathrm{MPa}$ corresponds to a change in the compressibility by this transition, $f_{0} \Delta K(\mathrm{~S}-$ T), which is apparently negative. This result means that the compressibility is decreased by the transition from the $\mathrm{S}$ state to the $\mathrm{T}$ state. In this case, we obtained $K_{0}=-13 \mathrm{~cm}^{3} \mathrm{~mol}^{-1}, K_{1}=(2.2 \pm 0.8) \times 10^{-2} \mathrm{~cm}^{3}$ $\mathrm{mol}^{-1} \mathrm{MPa}^{-1}, K_{2}=-(2.2 \pm 0.5) \times 10^{-4} \mathrm{~cm}^{3} \mathrm{~mol}^{-1} \mathrm{MPa}^{-2}$. From this fitting curve, we obtained the volume change and compressibility change at $P=0.1$ as $f_{0} \Delta V(\mathrm{~S}-\mathrm{T})=K_{0}=-13 \mathrm{~cm}^{3} \mathrm{~mol}^{-1}$ and $f_{0} \Delta K(\mathrm{~S}-\mathrm{T})=-K_{1}$ $=-2.2 \times 10^{-2} \mathrm{~cm}^{3} \mathrm{~mol}^{-1} \mathrm{MPa}^{-1}$. Hereafter we use these values.

3.3 Compressibility Changes of the $\mathbf{D} \rightarrow$ T Process. The pressure dependence of the volume difference between the initial and final states $(\Delta V(\mathrm{D}$-fin $))$ was measured by the $\operatorname{TrL}$ method. It should be noted here that the final reaction product is a mixture of the $\mathrm{T}$ state of the reactive form and the $\mathrm{S}$ state of the non-reactive form. The TrL signals from the phot1LOV2-linker and the calorimetric reference sample over a wide time range is shown in Fig. 7(a). 
Fig. 7
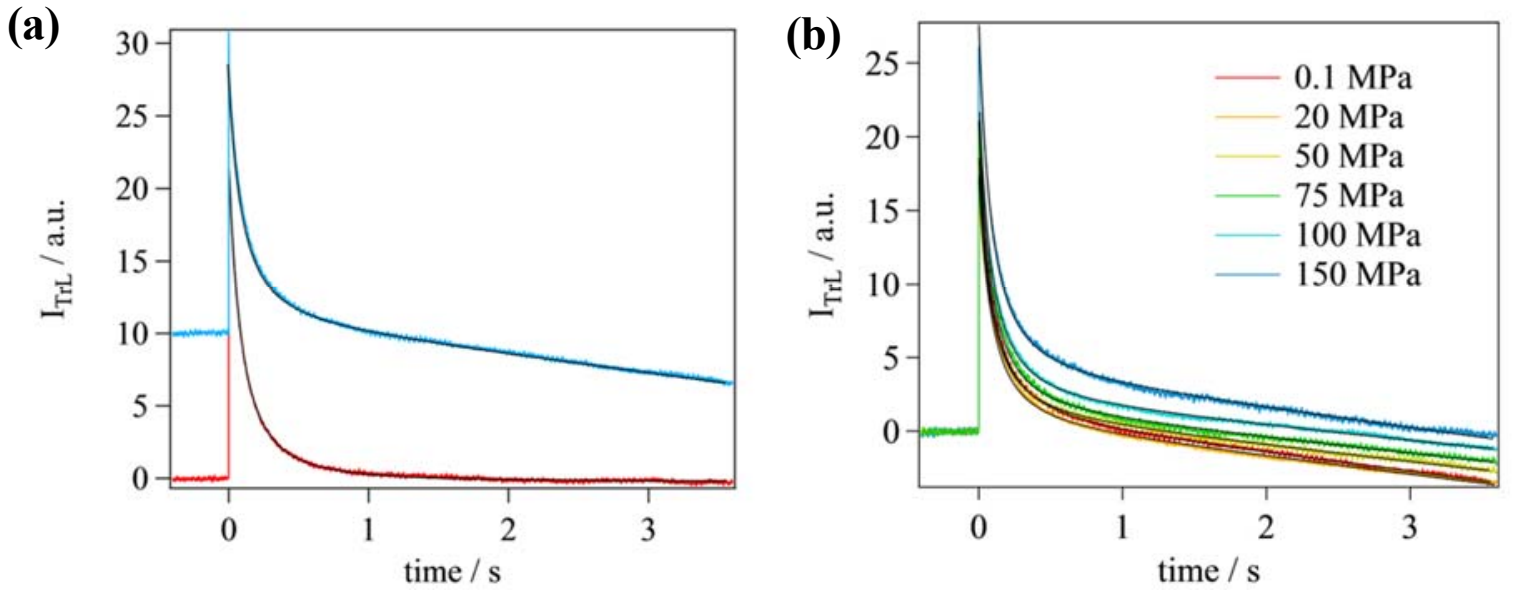

Figure 7. (a) TrL signals of the phot1LOV2-linker (blue line) and of the calorimetric reference sample (red line) at ambient pressure. The signal of the phot1LOV2-linker was shifted vertically to avoid overlap. The best fitted curves are shown by the black lines. (b) Pressure dependence of the TrL signal of the phot1LOV2-linker. Pressures are indicated by the legend in the figure.

The initial decay component over the $0-300 \mathrm{~ms}$ range is the thermal lens signal mentioned in the above section. The subsequent decaying component for the phot1LOV2-linker represents the diffusion processes of the reactant and final product. Since this time constant is very long (in the order of $100 \mathrm{~s}$ ) compared with this time range, this part can be approximated by a linear function of time. Hence, the signal was fitted by:

$$
I_{\operatorname{TrL}}(\mathrm{t})=\beta\left\{\delta n^{\prime}{ }_{\mathrm{th}}(t)+\left(\left(\delta n_{\mathrm{fin}}-\delta n_{\mathrm{D}}\right)\left(1-A^{\prime} t\right)\right\}\right.
$$

where $A^{\prime}$ is a constant. The temporal profile of the thermal lens signal $\delta n^{\prime}$ th $(t)$ was determined from the signal of the calorimetric reference sample. Here, $\delta n_{\text {fin }}$ denotes the refractive index change of the final 
state. There are two contributions in $\delta n_{\text {fin; }}$ the $\mathrm{T}$ state of the reactive form and the S state of the nonreactive form. Since the absorption spectrum changes with the transition from the D to $\mathrm{S}$ state, the amplitude of the refractive index, $\left(\delta n_{\mathrm{fin}}-\delta n_{\mathrm{D}}\right)$ should be the sum of the population lens $\left(\delta n_{\text {pop }}\right)$ and the volume lens $\left(\delta n_{\mathrm{V}}\right)$ contributions (SI-1). However, this population lens term does not depend on the pressure because the absorption spectrum does not depend on the pressure. Therefore, by taking the pressure dependent component in $\left(\delta n_{\text {fin }}-\delta n_{\mathrm{D}}\right)$, we can extract the pressure dependent volume contribution:

$$
\Delta P\left(\delta n_{\mathrm{fin}}-\delta n_{\mathrm{D}}\right)=\Delta \mathrm{P}\left(\delta n_{\mathrm{V}}+\delta n_{\mathrm{pop}}\right)=\Delta \mathrm{P} \delta n_{\mathrm{V}}
$$

where $\Delta_{P}$ means the difference against $P$. The volumetric quantity extracted from this relationship is expressed by $\Delta_{P} \Delta V$ (D-fin), which means the pressure-induced change in the volume difference between the final and dark states $(\Delta V(\mathrm{D}$-fin $))$. From the pressure dependence of the TrL signal shown in Fig. 7(b), ${ }_{P} \Delta V(\mathrm{D}$-fin) against the pressure was obtained and is presented in Fig. 8.

Fig. 8

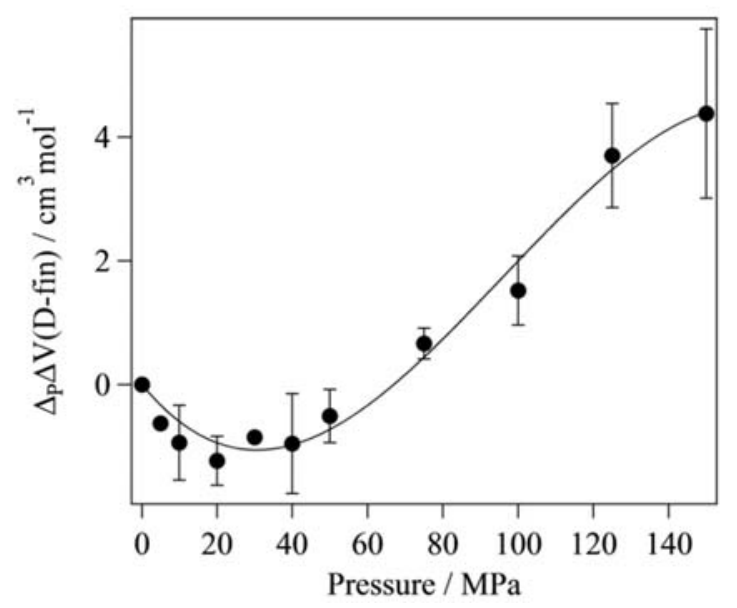


Figure 8. Pressure dependence of the volume change associated with the transition from the D state to the T state (circles). The best fit by Eq. (8) is shown by the smooth curve.

Note that this quantity contains contributions from the $\mathrm{S}$ state of non-reactive species and the T state of reactive species.

It is interesting to find that $\Delta V$ (D-fin) initially decreased and then increased at $\sim 20 \mathrm{MPa}$. We fitted the data within the range of 0.1 to $150 \mathrm{MPa}$ by:

$$
\Delta_{\mathrm{P}} \Delta V(\mathrm{D} \text {-fin })=\Delta K_{1}(\mathrm{P}-0.1)+\Delta K_{2}(\mathrm{P}-0.1)^{2}+\Delta K_{3}(\mathrm{P}-0.1)^{3}
$$

The best fit curve is shown in Fig. 8. From this fitting, we determined $\Delta K_{1}=-(7.4 \pm 0.9) \times 10^{-2}$ $\mathrm{cm}^{3} \mathrm{MPa}^{-1}, \Delta K_{2}=(1.4 \pm 0.2) \times 10^{-3} \mathrm{~cm}^{3} \mathrm{MPa}^{-3}, \Delta K_{3}=-(5.0 \pm 1) \times 10^{-6} \mathrm{~cm}^{3} \mathrm{MPa}^{-1}$. The compressibility change for the final state at ambient pressure is given by the pressure derivative of Eq. (8) at $P=0.1 \mathrm{MPa}$ (i.e., $\left.-\Delta K_{1}\right) ; \Delta K(\mathrm{D}$-fin $)=(7.4 \pm 0.9) \times 10^{-2} \mathrm{~cm}^{3} \mathrm{MPa}^{-1}$.

This compressibility change is a sum of those from the reactive and non-reactive forms. We separated these contributions as follows. First, the apparent volume change $\Delta V(\mathrm{D}$-fin $)$ is divided into these two contributions:

$$
\Delta V(\mathrm{D} \text {-fin })=f(\mathrm{P}) \Delta V(\mathrm{D}-\mathrm{T})+(1-f(\mathrm{P})) \Delta V(\mathrm{D}-\mathrm{S})
$$

where $\Delta V(\mathrm{D}-\mathrm{T})$ and $\Delta V(\mathrm{D}-\mathrm{S})$ indicate the volume changes during the transition from the $\mathrm{D}$ to $\mathrm{T}$ states and from the D to S states, respectively; that is, the first and the second terms on the right hand side represent the contribution of the reactive and non-reactive forms, respectively. (Since the non-reactive 
form yields the $\mathrm{S}$ state but does not produce the $\mathrm{T}$ state, the volume change is given by $\Delta V(\mathrm{D}-\mathrm{S})$.) Furthermore, since $\Delta V(\mathrm{D}-\mathrm{T})$ should be a sum of $\Delta V(\mathrm{D}-\mathrm{S})$ and $\Delta V(\mathrm{~S}-\mathrm{T})(\Delta V(\mathrm{D}-\mathrm{T})=\Delta V(\mathrm{D}-\mathrm{S})+\Delta V(\mathrm{~S}-\mathrm{T}))$, Eq. (9) can be rewritten as:

$$
\Delta V(\mathrm{D} \text {-fin })=f(\mathrm{P}) \Delta V(\mathrm{~S}-\mathrm{T})+\Delta V(\mathrm{D}-\mathrm{S})
$$

Here, $\Delta V(\mathrm{~S}-\mathrm{T})$ is the volume change associated with the reaction from the $\mathrm{S}$ state to the $\mathrm{T}$ state; that is, the unfolding process of the linker domain. By taking pressure derivative of Eq. (10), one may obtain:

$$
\begin{aligned}
\Delta K(\mathrm{D} \text {-fin }) & =\left(\frac{\partial f(\mathrm{P})}{\partial \mathrm{P}}\right)_{T} \Delta V(\mathrm{~S}-\mathrm{T})+f(\mathrm{P}) \Delta K(\mathrm{~S}-\mathrm{T})+\Delta K(\mathrm{D}-\mathrm{S}) \\
= & 2.0 \times 10^{-3} f_{0} \Delta V(\mathrm{~S}-\mathrm{T})+\left(1-2.0 \times 10^{-3} P\right) f_{0} \Delta K(\mathrm{~S}-\mathrm{T})+\Delta K(\mathrm{D}-\mathrm{S})
\end{aligned}
$$

Here, we used the pressure dependent fraction of the reactive form $f(P)=f_{0}\left(1-2.0 \times 10^{-3} P\right)$ obtained before. Using $f_{0} \Delta V(\mathrm{~S}-\mathrm{T})=-13 \mathrm{~cm}^{3} \mathrm{~mol}^{-1}, f_{0} \Delta K(\mathrm{~S}-\mathrm{T})=-2.2 \times 10^{-2} \mathrm{~cm}^{3} \mathrm{~mol}^{-1} \mathrm{MPa}^{-1}$ and $\Delta K(\mathrm{D}-$ fin) $=7.4 \times 10^{-2} \mathrm{~cm}^{3} \mathrm{MPa}^{-1}$ at $P=0.1 \mathrm{MPa}$, we determined $\Delta K(\mathrm{D}-\mathrm{S})=0.12 \mathrm{~cm}^{3} \mathrm{MPa}^{-1}$ at ambient pressure. Furthermore, the compressibility change of the $\mathrm{T}$ species is given by:

$$
\Delta K(\mathrm{D}-\mathrm{T})=\Delta K(\mathrm{D}-\mathrm{S})+\Delta K(\mathrm{~S}-\mathrm{T})=0.12-0.022 / f_{0} \mathrm{~cm}^{3} \mathrm{~mol}^{-1} \mathrm{MPa}^{-1}
$$

As stated in section SI-5, $f_{0}$ is close to unity. The compressibility change along the reaction coordinate is depicted in Fig. 9 for $f_{0}=1$. 
Fig. 9

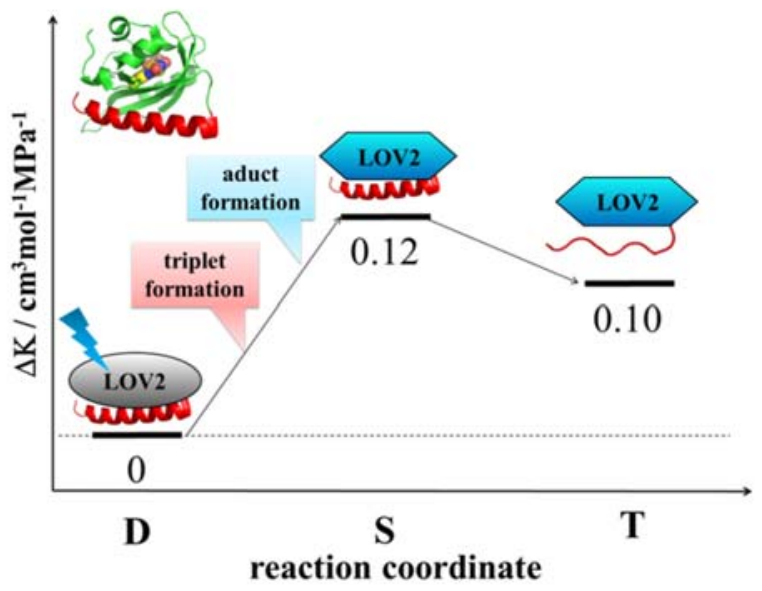

Figure 9. Schematic illustration of the volume fluctuation change from the dark state depicted along the reaction coordinate. For clarity, $f_{0}=1$ is used for this illustration.

\section{DISCUSSION}

As mentioned in the Introduction, the isothermal compressibility $\left(K_{\mathrm{T}}\right)$ reflects the volume fluctuation. In principle, the fluctuation determined from the compressibility includes the fluctuation of the protein and the solvated water molecules. However, this compressibility increase comes from the protein structure, because the conformation and solvation change detected by the $D$ change are not significant. One of most significant observations in this study is that we succeeded in detecting a transient enhancement of the isothermal compressibility (i.e., fluctuation) in the $\mathrm{S}$ state (and also in the $\mathrm{T}$ state compared with the D state) at ambient pressure. The observed enhancement of the compressibility relative to the dark state was $0.12 \mathrm{~cm}^{3} \mathrm{~mol}^{-1} \mathrm{MPa}^{-1}$. Although the compressibility in the dark state of the phot1LOV2-linker has not been reported, we roughly estimated the value as follow for comparison purposes. According to studies by Gekko et al., the square root of the volume fluctuation $\left(\sqrt{\left\langle(V-\langle V\rangle)^{2}\right\rangle}\right)$ of globular proteins is about $0.3 \%$ of their partial molar volume and the partial specific volumes of many globular proteins 
range from 0.7 to $0.75 \mathrm{~cm}^{3} \mathrm{~g}^{-1} \cdot{ }^{29,49}$ Using these data, the partial molar volume of the phot1LOV2-linker is estimated to be $\sim 19000 \mathrm{~cm}^{3} \mathrm{~mol}^{-1}$ assuming a partial specific volume of $0.75 \mathrm{~cm}^{3} \mathrm{~g}^{-1}$. Therefore, the phot1LOV2-linker square root of the volume fluctuation in the dark state is calculated to be $\sim 56 \mathrm{~cm}^{3}$ $\mathrm{mol}^{-1}$, which corresponds to $\sim 1.3 \mathrm{~cm}^{3} \mathrm{~mol}^{-1} \mathrm{MPa}^{-1}$. Using this value, the observed enhancement in the $\mathrm{S}$ state is an increase of $\sim 9.3 \%$ from the $\mathrm{D}$ state. This increase of $9.3 \%$ in the compressibility does not come from the whole protein, but is likely to be localized probably around an important region involved in the subsequent reaction (dissociation of the linker domain and unfolding of the helix) (vide infra). Hence, we consider that this is a relatively large enhancement and that this enhancement of the fluctuation can trigger the unfolding of the linker-helix. The extent of the enhancement of the compressibility $(\sim 9.3 \%)$ in this LOV domain sample is surprisingly similar to that of the BLUF domain protein, which was reported before $(\sim 10 \%)^{33}$ despite the difference in their fold and photoreaction. In the case of the BLUF domain, we considered that the increase of the fluctuation is localized in the interface region of the oligomer and triggers the dissociation of TePixD decamer. The similar extent of the increase of the fluctuation suggests that the dissociation of the LOV domain and the linker domain is also controlled by the fluctuation of the LOV domain.

The enhanced fluctuation of the light state of the LOV domain has been suggested previously. ${ }^{35}$ The solution NMR study of the LOV2-linker domain showed that the H-D exchange rate increases for the entire LOV core in the light state, indicating an increase in domain flexibility. ${ }^{35}$ Similarly, it was reported that the LOV domain of Brucella LOV-HK in the light state is more susceptible to proteolysis. ${ }^{9}$ These reports are consistent with our results in this study. We found that the fluctuation is enhanced already in the $\mathrm{S}$ state and decreases slightly in the final $\mathrm{T}$ state. We also presented a quantitative way to characterize the fluctuation.

An important contribution to the compressibility is the cavities inside the protein. ${ }^{49-51}$ A detailed description that explains the enhanced compressibility is obtained from the crystal structure of the 
phot1LOV2-linker of Avena sativa for dark and light states. ${ }^{7}$ Using the PDB data of 2V1A and 2V1B, which correspond to the dark and light structures, respectively, we calculated their cavities by the 3D $\operatorname{program}^{52}$ with a water probe radius of $1.5 \AA$. (Here 'cavity volume' was defined by the difference between the molecular volume and the van der Waals volume.) The light state of the crystal structure does not represent the structure of the T state, but it can approximately reflect the structure of the S state, because the crystal packing does not allow the large conformation change in the linker region. Hence one may consider that the light state structure in the crystal should resemble the S state structure, in which the conformation change is rather localized around the chromophore. We found that the cavity volume of the light state was larger than that of the dark state by $8 \mathrm{~cm}^{3} \mathrm{~mol}^{-1},\left(1707 \mathrm{~cm}^{3} \mathrm{~mol}^{-1}\right.$ for the light state and $1699 \mathrm{~cm}^{3} \mathrm{~mol}^{-1}$ for the dark state). Although this difference appears small, it is known that this change may be sufficient to explain the observed compressibility change as follows. It was reported that, in the case of $E$. coli dihydrofolate reductase, an increase of the cavity volume by $7.2 \mathrm{~cm}^{3} \mathrm{~mol}^{-1}$ resulted in an increase of the adiabatic compressibility by $0.11 \mathrm{~cm}^{3} \mathrm{~mol}^{-1} \mathrm{MPa}^{-1}{ }^{29}$ This observation agrees quantitatively well with our case; i.e., the increase of the cavity by $8 \mathrm{~cm}^{3} \mathrm{~mol}^{-1}$ results in an increase of the isothermal compressibility by $0.12 \mathrm{~cm}^{3} \mathrm{~mol}^{-1} \mathrm{MPa}^{-1}$.

The cavities inside the protein structures in the dark (2V1A) and light state (2V1B) are depicted in Fig. 10(a) and (b) by navy and yellow regions, respectively, superimposed onto the 2V1A structure.

Fig. 10 

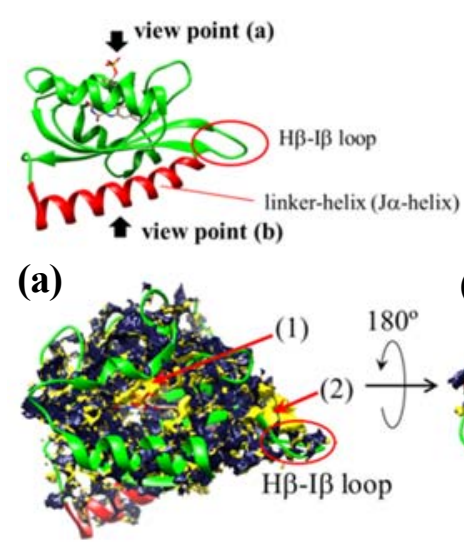

(b)

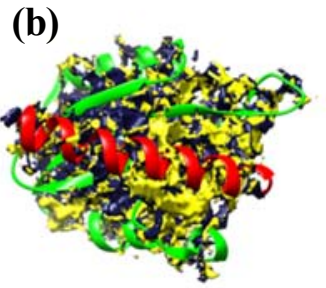

Figure 10. (Upper) Structure of the phot1LOV2-linker of Avena sativa (PDB ID: 2V1A). Black arrows indicate the direction from which the structure is drawn in (a) and (b). (Lower) (a) and (b) are the structures of the same protein seen from the viewpoint above and below the chromophore, respectively. Navy and yellow surfaces show the cavity area in the light and dark states, respectively.

The yellow dominant region indicates a region where the cavities are generated in the light state; that is, it is the more compressible region and hence would have a larger fluctuation in the light state. The yellow region is dominant in the interface region between the J- $\alpha$ helix (linker-helix) and LOV core (Fig. 10(b)) than in the upper side of the LOV domain (Fig. 10(a)). This result implies that the enhanced fluctuation is localized to this interface region, which eventually triggers the dissociation of the linker domain from the LOV domain. In Fig. 10(a), there are two distinct yellow rich regions indicated by (1) and (2). Region (1) is part of the hydrophobic flavin binding pocket. It has been pointed out that some residues around the chromophore move upon the adduct formation. ${ }^{7,9,11,35}$ Such movements may create the cavity observed as region (1). Region (2) is located near the H $\beta$-I $\beta$ loop. Indeed, molecular dynamic simulations of the phot1LOV2 domain showed that the fluctuation of this loop increases in the light state. ${ }^{10}$ On the basis of these considerations, we speculate that upon adduct formation, cavities are created in the binding pocket and this leads to incomplete packing of the LOV core, thereby allowing the H $\beta$-I $\beta$ 
loop and $\beta$-scaffold to fluctuate more strongly, which results in the dissociation of the linker helix.

Another interesting observation is that the compressibility slightly decreases upon transition from the $\mathrm{S}$ to $\mathrm{T}$ states. This decrease is reasonable, because the cavities around the linker-helix could collapse by the unfolding of the linker helix and hydration takes place subsequently owing to exposure of the interior. These changes cause a decrease in the volume fluctuation in the T state. Indeed, a large decrease in $K_{\mathrm{T}}$ by the unfolding of globular proteins has been reported $\left(-24 \mathrm{Mbar}^{-1}\right.$ for lysozyme, ${ }^{53}-30 \mathrm{Mbar}^{-1}$ for Ribonuclease $\mathrm{A}^{54}$ ) and it was explained by the collapse of internal cavities and increase in hydration upon exposure of the interior. ${ }^{53,54}$ On the other hand, the decrease in the compressibility by the $\mathrm{S} \rightarrow \mathrm{T}$ transition is $-2.2 \times 10^{-2} \mathrm{~cm}^{3} \mathrm{~mol}^{-1} \mathrm{MPa}^{-1}$ (for $f_{0}=1$ ) and this change corresponds to a $1.7 \%$ decrease of the fluctuation of the dark state. Taking into consideration the number of residues that form the linker region $(586 \mathrm{R}-661 \mathrm{~K}, 75$ residues) is $35 \%$ of the total number of the phot1LOV2-linker $(449-661 \mathrm{~K}, 212$ residues), we believe that the compressibility decrease of $1.7 \%$ is very small. This discrepancy indicates that the unfolding of the linker may not be a complete unfolding event, whereas previous studies used $\mathrm{GuHCl}$ as a denaturant to completely unfold the proteins.

\section{CONCLUSIONS}

The pressure effect on the photochemical reaction of the phot1LOV2-linker was investigated by optical absorption spectroscopy and the time-resolved TG and $\operatorname{TrL}$ methods in the time domain. Our high pressure optical cell enabled us to compare the TG signal amplitudes of different samples quantitatively, and we studied the pressure effects on the thermodynamic properties of the reaction intermediates. The pressure dependence of the absorption spectrum indicates that the conformation is stable against pressure. Interestingly, larger compressibility for the S species was observed when compared with the dark state. The increased compressibility indicates that volume fluctuations for these intermediates are enhanced. The cavity map of the LOV domain suggests that this enhancement comes from the cavities around the 
LOV domain-linker interface region and this triggers the dissociation reaction of the linker part. The detailed analysis also revealed the compressibility of the $\mathrm{T}$ state and it was found that the compressibility decreases during the transition from the $\mathrm{S}$ state to $\mathrm{T}$ state. This decrease was explained by the collapse of cavities of the linker helix following its unfolding. Although there are no experimental data showing enhanced fluctuation of intermediate species during the reaction, except for TePixD, ${ }^{33}$ such indications have been reported for some protein systems. The solution NMR study of the LOV2-linker domain showed that the H-D exchange rate is increased for the entire LOV core in the light state, indicating an increase in domain flexibility. ${ }^{35}$ Additionally, the light state of the LOV domain of Brucella is more susceptible to proteolysis. ${ }^{9}$ MD simulations studies on the LOV domain showed an increase in the fluctuations of its H $\beta$-I $\beta$ loop region in the light state. ${ }^{10,11}$ The structurally related PAS (PerArntSim) domain protein PYP (photoactive yellow protein), whose N-terminal cap dissociates from the central $\beta$ sheet and unfolds upon reaching the signaling state ${ }^{55}$, was reported to be more susceptible to mechanical forces in the light state, suggesting that PYP may adopt a less compact structure in this state. ${ }^{56}$ This observation can also be interpreted as the PYP protein showing larger fluctuations in the light state. Since the signaling mechanism is similar to that of the LOV domain, ${ }^{57}$ PYP may also employ structural fluctuations to facilitate its reaction and such a reaction mechanism may be a common characteristic of the PAS domain. The present study should be important for making a concept of importance role of fluctuation in the reaction.

\section{ACKNOWLEDGMENTS}

This work was supported by a Grant-in-aid for Scientific Research on Innovative Areas (research in a proposed research area) (Nos. 20107003, and 25102004) and a Grant-in-aid for Scientific Research (25288005) from the Ministry of Education, Culture, Sports, Science and Technology in Japan (to M.T.). 


\section{REFERENCES}

1. A. Pudasaini, K. K. El-Arab and B. D. Zoltowski, Front. Mol. Biosci., 2015, 2, 18.

2. A. Losi and W. Gartner, Annu. Rev. Plant. Biol., 2012, 63, 49-72.

3. T. Kottke, P. Hegemann, B. Dick and J. Heberle, Biopolymers, 2006, 82, 373-378.

4. S. Crosson, S. Rajagopal and K. Moffat, Biochemistry, 2002, 42, 2-10.

5. Y. Nakasone, T. Eitoku, D. Matsuoka, S. Tokutomi and M. Terazima, Biophys. J., 2006, 91, 645653.

6. T. Bednarz, A. Losi, W. Gartner, P. Hegemann and J. Heberle, Photochem. Photobiol. Sci., 2004, 3, 575-579.

7. A. S. Halavaty and K. Moffat, Biochemistry, 2007, 46, 14001-14009.

8. A. Pfeifer, T. Majerus, K. Zikihara, D. Matsuoka, S. Tokutomi, J. Heberle and T. Kottke, Biophys. J., 2009, 96, 1462-1470.

9. J. Rinaldi, M. Gallo, S. Klinke, G. Paris, H. R. Bonomi, R. A. Bogomolni, D. O. Cicero and F. A. Goldbaum, J. Mol. Biol., 2012, 420, 112-127.

10. P. L. Freddolino, M. Dittrich and K. Schulten, Biophys. J., 2006, 91, 3630-3639.

11. P. L. Freddolino, K. H. Gardner and K. Schulten, Photochem. Photobiol. Sci., 2013.

12. E. Peter, B. Dick and S. Baeurle, J. Mol. Model., 2012, 18, 1375-1388.

13. M. Karplus and G. A. Petsko, Nature, 1990, 347, 631-639.

14. V. A. Feher and J. Cavanagh, Nature, 1999, 400, 289-293.

15. H. Yang, G. Luo, P. Karnchanaphanurach, T.-M. Louie, I. Rech, S. Cova, L. Xun and X. S. Xie, Science, 2003, 302, 262-266.

16. K. Akasaka, Biochemistry, 2003, 42, 10875-10885.

17. S. Y. Noskov, S. Berneche and B. Roux, Nature, 2004, 431, 830-834.

18. K. Gekko, N. Obu, J. Li and J. C. Lee, Biochemistry, 2004, 43, 3844-3852. 
19. G. Bhabha, J. Lee, D. C. Ekiert, J. Gam, I. A. Wilson, H. J. Dyson, S. J. Benkovic and P. E. Wright, Science, 2011, 332, 234-238.

20. T. Kosugi and S. Hayashi, J. Am. Chem. Soc., 2012, 134, 7045-7055.

21. K. Henzler-Wildman and D. Kern, Nature, 2007, 450, 964-972.

22. T. Eitoku, Y. Nakasone, K. Zikihara, D. Matsuoka, S. Tokutomi and M. Terazima, J. Mol. Biol., 2007, 371, 1290-1303.

23. N. Baden, S. Hirota, T. Takabe, N. Funasaki and M. Terazima, J. Chem. Phys., 2007, 127, 175103.

24. K. Inoue, J. Sasaki, M. Morisaki, F. Tokunaga and M. Terazima, Biophys. J., 2004, 87, 2587-2597.

25. M. Terazima, J. Photochem. Photobiol., C, 2002, 3, 81-108.

26. M. Sakakura, S. Yamaguchi, N. Hirota and M. Terazima, J. Am. Chem. Soc., 2001, 123, 42864294.

27. T. V. Chalikian, Annu. Rev. Biophys. Biomol. Struct., 2003, 32, 207-235.

28. N. Taulier and T. V. Chalikian, Biochim. Biophys. Acta, 2002, 1595, 48-70.

29. K. Gekko, Biochim. Biophys. Acta, 2002, 1595, 382-386.

30. T. V. Chalikian, M. Totrov, R. Abagyan and K. J. Breslauer, J. Mol. Biol., 1996, 260, 588-603.

31. A. Cooper, Proc. Natl. Acad. Sci. U. S. A., 1976, 73, 2740-2741.

32. Y. Hoshihara, Y. Kimura, M. Matsumoto, M. Nagasawa and M. Terazima, Rev. Sci. Instrum., 2008, 79, 034101.

33. K. Kuroi, K. Okajima, M. Ikeuchi, S. Tokutomi and M. Terazima, Proc. Natl. Acad. Sci. U. S. A., 2014, 111, 14764-14769.

34. J. M. Christie, Annu. Rev. Plant. Biol., 2007, 58, 21-45.

35. S. M. Harper, L. C. Neil and K. H. Gardner, Science, 2003, 301, 1541-1544.

36. Y. Nakasone, T. Eitoku, D. Matsuoka, S. Tokutomi and M. Terazima, J. Mol. Biol., 2007, 367, $432-442$. 
37. T. Eitoku, Y. Nakasone, D. Matsuoka, S. Tokutomi and M. Terazima, J. Am. Chem. Soc., 2005, $127,13238-13244$

38. M. Terazima, Biochim. Biophys. Acta, 2011, 1814, 1093-1105.

39. Y. Nakasone, T. Eitoku, K. Zikihara, D. Matsuoka, S. Tokutomi and M. Terazima, J. Mol. Biol., 2008, 383, 904-913.

40. M. Kasahara, T. E. Swartz, M. A. Olney, A. Onodera, N. Mochizuki, H. Fukuzawa, E. Asamizu, S. Tabata, H. Kanegae, M. Takano, J. M. Christie, A. Nagatani and W. R. Briggs, Plant Physiol., 2002, 129, 762-773.

41. K. Takeda, Y. Nakasone, K. Zikihara, S. Tokutomi and M. Terazima, J. Phys. Chem. B, 2013, 117, 15606-15613.

42. M. Nakasako, T. Iwata, D. Matsuoka and S. Tokutomi, Biochemistry, 2004, 43, 14881-14890.

43. M. Terazima, Phys Chem Chem Phys, 2006, 8, 545-557.

44. M. Terazima, in Advances in Photochemistry, eds. D. C. Neckers, D. H. Volman and G. V. Bünau, John Wiley \& Sons, Inc., 2007, vol. 24, pp. 255-338.

45. M. Terazima and N. Hirota, J. Phys. Chem., 1992, 96, 7147-7150.

46. M. Terazima, Isr. J. Chem., 1998, 38, 143-157.

47. B. B. Boonyaratanakornkit, C. B. Park and D. S. Clark, Biochim. Biophys. Acta, 2002, 1595, 235249.

48. K. Kuroi, K. Okajima, M. Ikeuchi, S. Tokutomi, T. Kamiyama and M. Terazima, J. Phys. Chem. $B, 2015,119,11$.

49. K. Gekko and Y. Hasegawa, Biochemistry, 1986, 25, 6563-6571.

50. T. Kamiyama and K. Gekko, Biochim. Biophys. Acta, 2000, 1478, 257-266.

51. S. Mimura, T. Yamato, T. Kamiyama and K. Gekko, Biophys. Chem., 2012, 161, 39-45.

52. N. R. Voss and M. Gerstein, Nucleic Acids Res, 2010, 38, W555-W562. 
53. T. Kamiyama and K. Gekko, Chem. Lett., 1997, 26, 1063-1064.

54. Y. Tamura and K. Gekko, Biochemistry, 1995, 34, 1878-1884.

55. M. Harigai, Y. Imamoto, H. Kamikubo, Y. Yamazaki and M. Kataoka, Biochemistry, 2003, 42, 13893-13900.

56. J. M. Zhao, H. Lee, R. A. Nome, S. Majid, N. F. Scherer and W. D. Hoff, Proc. Natl. Acad. Sci. U. S. A., 2006, 103, 11561-11566.

57. D. Hoersch, F. Bolourchian, H. Otto, M. P. Heyn and R. A. Bogomolni, Biochemistry, 2010, 49, 10811-10817. 


\section{TOC graphic}

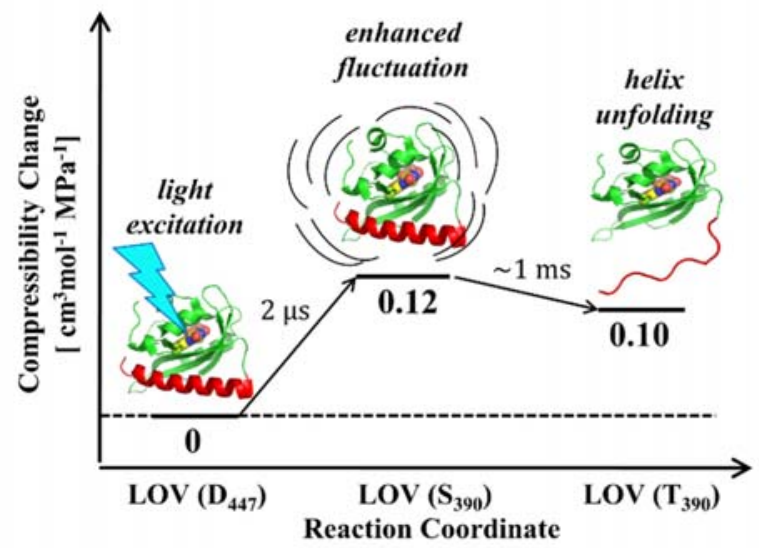

